\title{
Novel Imaging Radar Technology for Detection of Landmines and Other Unexploded Ordnance
}

\author{
Markus Peichl $^{1}$ (D) Eric Schreiber ${ }^{1} \cdot$ Andreas Heinzel $^{1} \cdot$ \\ Stephan Dill ${ }^{1}$
}

Received: 9 August 2016/Accepted: 26 October 2016/Published online: 7 November 2016

(C) Springer International Publishing Switzerland 2016

\begin{abstract}
The safe, reliable, and efficient detection and subsequent removal of buried landmines or other unexploded ordnance (UXO) still remains a challenge. According to Landmine Monitor (2015), almost 60 states suffer the threat of antipersonnel mine contamination, while the total extent of contaminated areas is likely to be thousands of square kilometers and the total number of threat objects may be well over 100 million. In contrast, total annual clearing rates are in the order of about 230,000 landmines and about $200 \mathrm{~km}^{2}$ for the year of 2014, as the number of new contaminations continues to grow. Although non-technical surveillance for the identification of contamination improves slowly, more progress is needed in the basic detection of UXO. Although many new technologies have been investigated the last 20 years, the classical ones like metal detectors and dogs in cooperation with humans operators are still the most commonly ones used today. Hence, the detection process for many scenarios is unacceptably slow and dangerous for the operators, as they are typically less than $1 \mathrm{~m}$ away from the threats. Hence, a sensor technology enabling a sufficient stand-off distance for operators and reliable detection at high area throughput is desirable. The following paper describes the physical background, the system design, and representative measurement results of our innovative radar approach to this problem.
\end{abstract}

Keywords Security research - Landmine detection - Unexploded ordnance · Synthetic aperture radar - Spatial resolution · Radar clutter

Markus Peichl

markus.peichl@dlr.de

1 Deutsches Zentrum für Luft- und Raumfahrt (DLR), Institut für Hochfrequenztechnik und Radarsysteme, Münchener Straße 20, 82234 Weßling, Germany 


\section{Introduction}

The reliable detection of buried UXO in earth ground is a complex undertaking, especially when the articulation of the scene to be investigated can have many facets. There are thousands of possible target types comprising many different materials and constructions, buried in very different types of earth and covered by various types of vegetation or obscurants. Access also becomes an additional problem, potentially arising due to natural or man-made activity. However, many new and different sensing approaches have been investigated in the past 20 years, e.g., infrared sensing of small temperature gradients, laser vibrometry using acoustic ground stimulation, training of rats and bees for detecting explosive vapor based on an awarding principle, electronic devices for reacting on explosive vapor by changing electrical currents, Terahertz spectroscopy to measure typical explosive fingerprint spectra, bacteria to become fluorescent by explosive digestion, and ground penetrating radars (GPR) either close to ground or stand-off to detect impedance mismatches and backscatter differences in ground, just to mention some of those. On the other hand, the classical methods like metal detectors or prodders, the latter being a type of fumbling rods, have been improved as well to increase detection capabilities. Besides new sensing technologies, the use of robots and remotely controlled vehicles as sensor platforms has been advanced to reduce risk of the deminers. In addition, brute force methods like soil pronging tanks or plants are available, being used predominantly for fast military clearing. Figure 1 illustrates few of the above mentioned methods and technologies for better understanding.
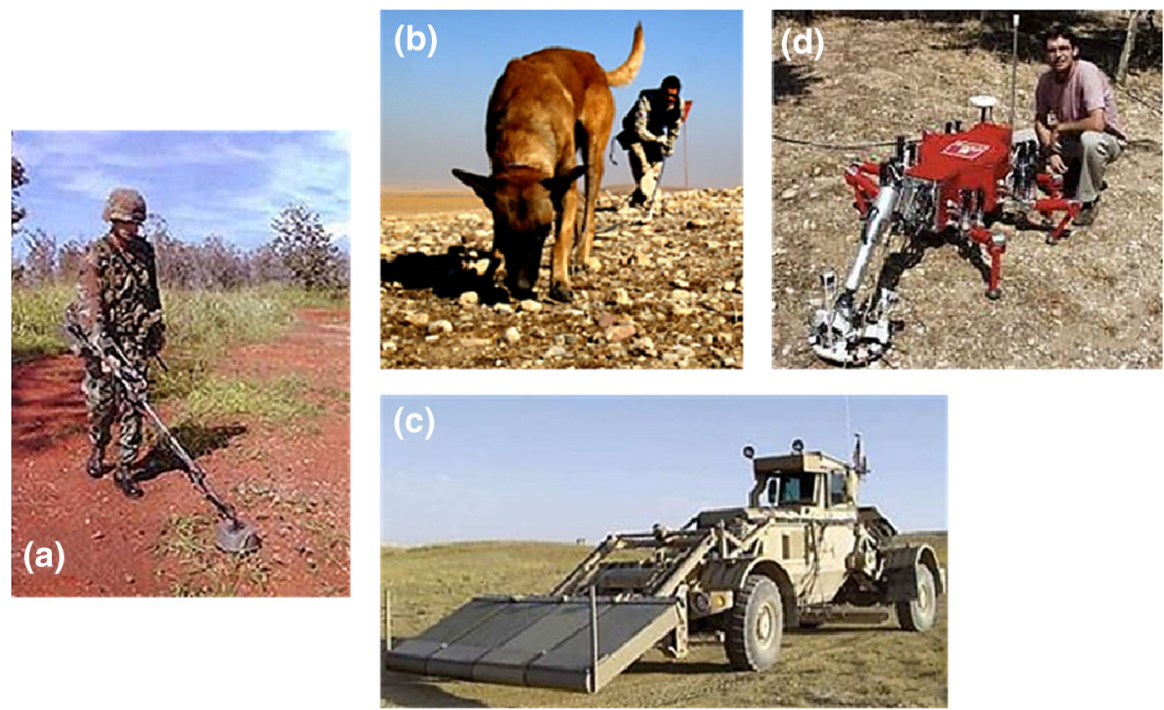

Fig. 1 Photographs of various methods used for landmine and UXO detection; a manually operated combination of metal detector and GPR (Pica.Army 2016); b mine detection dog in action (Globalgiving 2016); c GPR array carried by a military vehicle (Theleadsouthaustralia 2016); d GPS assisted robot with a sensor embedded head to detect landmines (Telovation 2016) 
Basically, humanitarian demining and military demining have to be differentiated, since the situation when and how to clean and the tolerable level of residual risk due to remaining threats, are different. However, most methods and technologies are similar or even identical for both cases. During the past years of, it became evident that no technology on its own can solve the problem of safe, reliable and efficient detection. Hence, the most suitable approach will be the use of a multi-sensor system and a certain degree of data fusion, which could improve reliability considerably. However, the safety and efficiency of the demining process is not directly addressed by that. Most practiced methods today operate rather close to the threats and in a rather punctual measurement mode, which makes demining dangerous for the operators and provides low aerial throughput with time. Consequently, a system operating as illustrated in Fig. 2 can fill this gap.

Here the sensor is moving parallel to and along the contaminated area in sufficiently safe distance on safe ground. Using, for instance, a ground vehicle, the sensor is elevated above the scene to several meters and the stand-off range should be in the order of several meters, providing sufficient slant visibility on the contaminated area. The width of the actually observed contaminated area should be in the order of several meters, and the length is defined by the selected path length of the moving sensor. Such sensors are optimized for maximum and reliable detection performance of all objects in the scene, having not to discriminate in situ between true threats and false targets. Once a preferably geo-referenced detection map has been created, only the locations of detections need to be investigated further by applying a local suite of specific metal, chemical, GPR sensors in close proximity. The close-in secondary sensors can also be mounted on a steerable ground-based vehicle and optimized for detection and confirmation.

However, it is evident that a single system is not optimum for every scenario. The differing scenarios of a dense forest or jungle, rice field areas, densely covered urban structures, or strongly ragged terrain, will require specifically tuned sensor configurations. Nevertheless, there are many contaminated areas where the proposed approach can deliver considerable added value. A very suitable sensor type for that task is synthetic aperture radar (SAR). SAR techniques for landmine

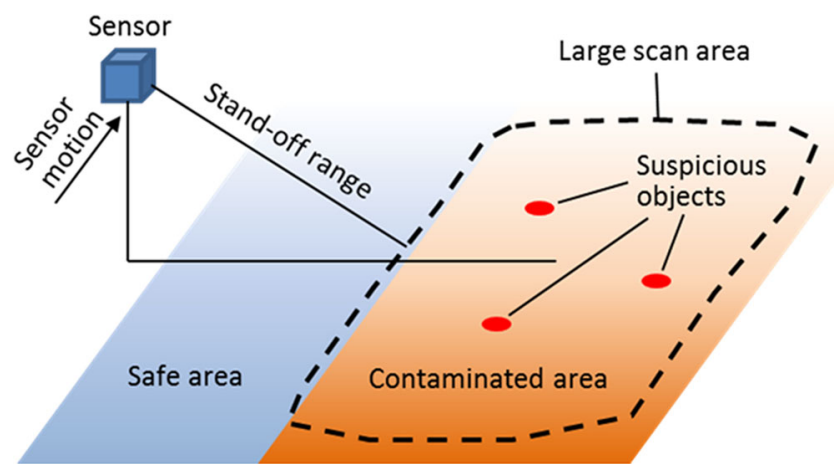

Fig. 2 Ideal sensing approach for an advanced landmine and UXO detection system, operating from safe distance and scanning a large area in short time 
detection have been refined over a number of years (Lawrence et al. 1999; Liu et al. 2003; Lloyd and Longstaff 2003; Feng et al. 2005; Wu et al. 2010; Kabourek et al. 2012).

\section{Basics of Imaging Microwave Radar}

The interaction of electromagnetic waves with matter is dictated by the electric and magnetic properties of the material. The bulk is defined in terms of reflection, transmission, and absorption of incident energy. Measuring such parameters by sensors can deliver information about an object. A suitable frequency range to perform such observations with respect to sensor size, interaction responsivity, and hardware availability is the microwave region. Here the interaction is purely defined on the bulk behavior of the material, i.e., that determined by the permittivity, and the permeability of the material. Spectral line features generally appear in the terahertz, infrared, and optical region of the electromagnetic spectrum, but radiation in those bands has insufficient penetration for buried UXO detection. Microwaves, on the other hand, with their longer wavelengths have sufficient penetration in and through many non-metallic materials, so buried objects with some reflectivity will generate a signature. Hence in theory, even objects buried in natural soils can interact with incident waves, thus providing information on them. The microwave frequency range is roughly defined by $1-30 \mathrm{GHz}$, and the range of $30-300 \mathrm{GHz}$ is called the millimeter-wave range. Corresponding wavelengths are $30-1 \mathrm{~cm}$ and 10-1 mm. Detailed information on radar and electromagnetic waves can be found in Ulaby (1981), (1982).

\subsection{Reflection and Scattering}

Any material can be characterized by its complex permittivity $\varepsilon$ and its complex permeability $\mu$, both together defining the complex wave impedance $Z$ :

$$
\begin{gathered}
\varepsilon=\left(\varepsilon^{\prime}+j \varepsilon^{\prime \prime}\right) \varepsilon_{0}, \quad \mu=\left(\mu^{\prime}+j \mu^{\prime \prime}\right) \mu_{0}, \quad Z=\sqrt{\frac{\mu}{\varepsilon}, \quad j=\sqrt{-1} \quad(1 \mathrm{a}-\mathrm{c})} \\
\varepsilon_{0} \approx 8.854 \times 10^{-12} \frac{A s}{V m}, \quad \mu_{0} \approx 1.257 \times 10^{-6} \frac{V s}{A m}, \quad Z_{0}=\sqrt{\frac{\mu_{0}}{\varepsilon_{0}}} \approx 377 \quad(2 \mathrm{a}-\mathrm{c})
\end{gathered}
$$

The quantities $\varepsilon^{\prime}$ and $\varepsilon^{\prime \prime}$ are real and imaginary part of the intrinsic permittivity, and $\mu^{\prime}$ and $\mu^{\prime \prime}$ are real and imaginary part of the intrinsic permeability, both being exclusive material parameters to be in general determined by measurements. The other quantities $\varepsilon_{0}, \mu_{0}$ and $Z_{0}$ are the permittivity, permeability and wave impedance of vacuum or free space, being valid as well for normal atmospheric conditions of air. Now each abrupt change of wave impedance gives rise to certain wave reflection. For perpendicular incidence on a smooth interface between two materials, indexed by numbers 1 and 2, the field reflection coefficient is given by 


$$
r=\frac{Z_{1}-Z_{2}}{Z_{1}+Z_{2}}, \quad 0 \leq|r| \leq 1,
$$

where permittivity, permeability and wave impedance for each material are computed according to Eqs. $(1 \mathrm{a}-\mathrm{c})$ and $(2 \mathrm{a}-\mathrm{c})$. Note that, if material 2 is metal, the imaginary part of the intrinsic permittivity tends towards infinity, and thus the magnitude of the reflection coefficient approaches the value of $|r|=1$, i.e., a hundred percent reflection. Typical values for real and imaginary parts of the intrinsic permittivity of most natural materials are $\varepsilon^{\prime}=1-80$ and $\varepsilon^{\prime \prime}=0-40$ depending on various parameters like material composition, frequency, temperature, etc., where the maxima are valid for pure water. Most purely dielectric and partly transparent materials are much below the maxima in the order of up to 20 for the real part and up to 1 for the imaginary part. Furthermore, most natural materials as well as synthetics have no or very low magnetic features, and thus $\mu^{\prime}=1$ and $\mu^{\prime \prime}=0$ is a valid approximation.

Now the situation for a general imaging radar observing a landmine scene can be illustrated as shown in Fig. 3. Mono-static radar, i.e., the transmitter (TX) and the receiver $(\mathrm{RX})$ are located at the identical elevated position, produces a focused beam intersecting the ground with footprint area $A$. The beam is scanned in a suitable pattern as indicated by the dashed line across the scene to produce a radar image.

Now, if the ground would be homogeneous, the surface would be completely flat, and the beam had certain incidence angle with respect to the ground, most reflected

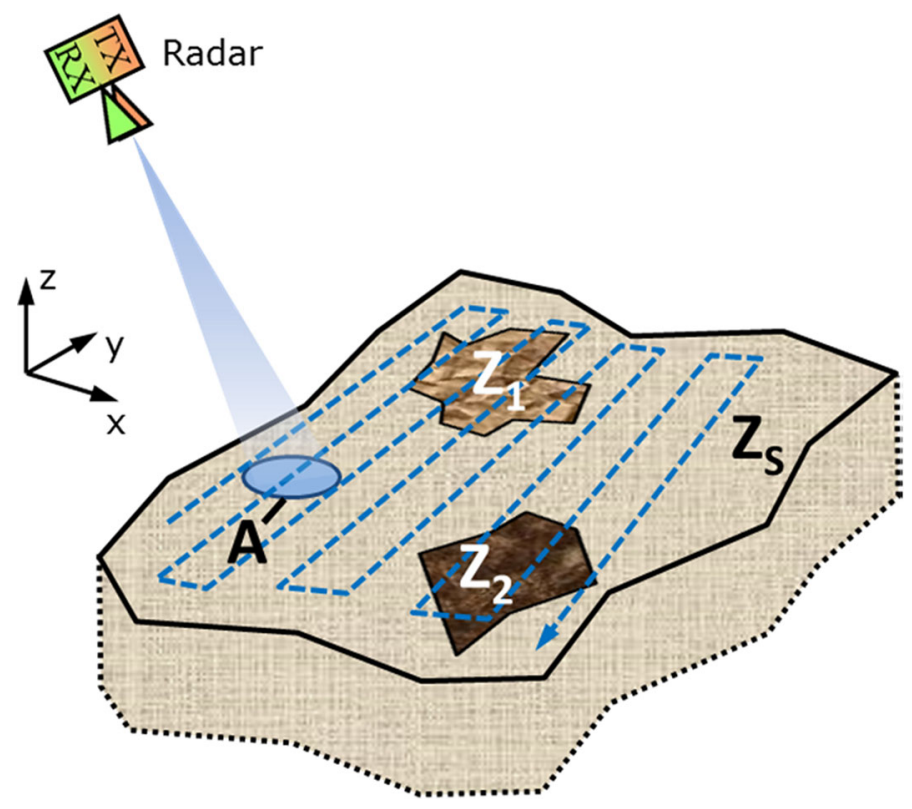

Fig. 3 Sketch of mono-static real-beam imaging radar scanning across a ground region. The ground has a general wave impedance $Z_{\mathrm{S}}$, and two target areas having different impedances $Z_{1}$ and $Z_{2}$ are embedded. The area of the focused antenna footprint is denoted by $A$ 
energy would be fully reflected in a direction away from the radar. So no information could be acquired. Fortunately, most natural surfaces are neither completely flat nor do they have no roughness. These two features cause, depending on their actual geometrical articulation that some energy is reflected back to the radar, called radar backscatter. The amount of backscatter cannot be described analytically due to the rather stochastic surface structure, but it is linked to the local changes in wave impedance between air and ground within the antenna footprint and across the whole scene. The quantity describing the backscatter of a surface, i.e., the backward reflected radiation, now is the radar cross-section (RCS) $\sigma$. The $\mathrm{RCS}$ is an equivalent area in square meters, usually being not identical to the observed footprint area, since it depends strongly on the specific structure and material type of the surface besides other physical parameters. Note that the RCS as well describes in the same way the backscatter of an isolated object within the antenna beam, e.g., a sphere, a cube, or a landmine, where for many canonical objects an analytical solution to compute the RCS exists. For natural surfaces, the RCS usually has to be measured and is expressed as the dimensionless RCS per unit area $\sigma^{0}$, so that the RCS of a certain area of size $A$ can be computed by

$$
\sigma=\sigma^{0} A \text {. }
$$

In general, the RCS of any object or surface depends in a complicated way on target parameters permittivity, permeability, shape and roughness, and from observation parameters frequency, incidence angle and polarization. Polarization characterizes the orientation of the incident electromagnetic fields with respect to the object or ground surfaces.

Consequently, by scanning a surface as indicated in Fig. 3, surface areas or even buried target areas can be detected by microwave radar purely based on mismatches in wave impedance, i.e., differences in permittivity and permeability between interfaces. However, those articulate weaker or stronger, heavily dependent also on previously mentioned other parameters, so that a few very important constraints on radar imaging have to be considered.

\subsection{Penetration Depth}

Since usually landmines and UXO are buried in the ground, it is of great importance to know how far radar can penetrate into soil and deliver useful information. The parameter describing quantitatively this ability is given by the penetration depth $d$,

$$
d=\sqrt{\varepsilon^{\prime}} /\left(2 \pi \varepsilon^{\prime \prime}\right)
$$

which is a definition based on power dissipation by a factor of $1 / e^{2}$ up to depth $d$, $e$ being Euler's constant, and $\lambda$ is the wavelength. Using a simple soil model to compute the permittivity of soil (Ulaby 1992) depending on moisture content, the penetration depth over frequency can be illustrated as shown in Fig. 4. Note that the permittivity due to the moisture content as well has a strong dependence on frequency. In summary it becomes evident that for a certain penetration capability into soil the lower microwave region has to be used. A reasonable upper value for 
Fig. 4 Penetration depth of microwaves in soil of different moisture content as a function of frequency. The yellow region is appropriate for buried object detection as in the case of demining (color figure online)
Soil of different moisture content

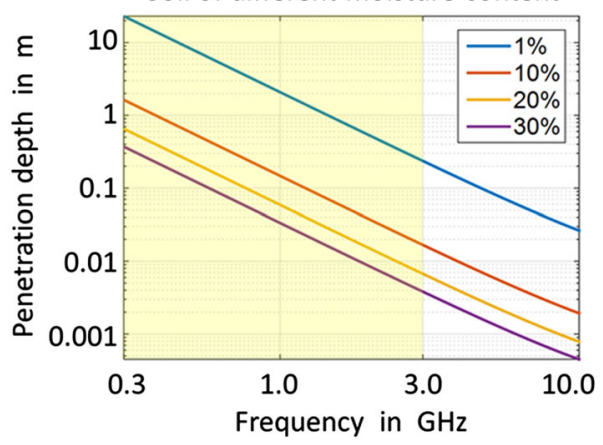

instance is $3 \mathrm{GHz}$ to achieve in most moisture conditions at least few centimeters of penetration. However, it should be emphasized that Eq. (5) is only a definition. Hence, the radar for buried object detection does not necessarily fail at larger depths or higher frequencies.

\subsection{Spatial Resolution}

The objects of interest as in the case of landmines and UXO are typically not larger than $30 \mathrm{~cm}$ diameter, e.g., anti-tank mines. Smallest objects like antipersonnel mines can be as small as $5 \mathrm{~cm}$ diameter, below that size the threat potential of any of such objects is too low, and the expense for resolving such targets in an image becomes unreasonably high. Figure 5 illustrates the extremal cases of very low and very high spatial resolution given in the size range of typical objects of interest.

It becomes evident that the spatial resolution of the imaging radar should achieve at least the size of the smallest objects to be detected. Otherwise, as shown in the low resolution image, targets may not be discriminated spatially from each other and the contrast with respect to the surrounding background decreases considerably.
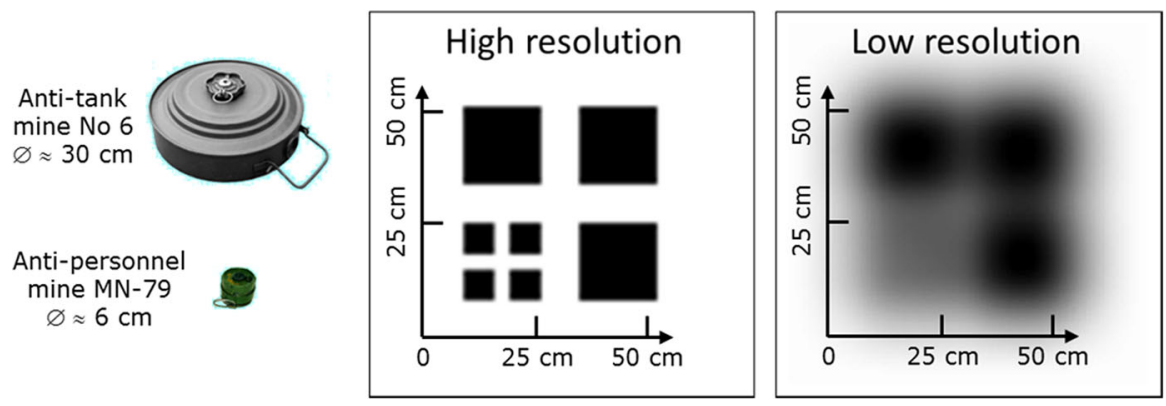

Fig. 5 Illustration of spatial resolution. The high resolution image shows three large and four small square-shaped objects imaged at high spatial resolution $<1 \mathrm{~cm}$; the low resolution image shows the same objects at low spatial resolution of $\sim 20 \mathrm{~cm}$; for comparison two real mines are shown in corresponding size to given $\mathrm{cm}$ scales 
Hence a successful detection may fail. A reasonable requirement for the spatial resolution to be achieved by the radar was estimated to be in the order of 5-10 cm.

\subsection{Backscatter of Targets and Background Clutter}

As mentioned previously, natural surfaces or backgrounds as present in the situation of landmine and UXO detection, e.g., grassland, meadows, raw soil, gravel, etc., can provide as well significant RCS. This circumstance is highly welcome for scientific Earth observation since it can provide information on ground characteristics like vegetation cover or soil moisture, for instance. In contrast, in case of surface and buried object detection, the background signatures, called clutter, can considerably hinder proper object detection. This situation can be illustrated by an optical counterpart as shown in Fig. 6.

In the optical image, a green ball is located on a green grass area. Although the ball can be made of completely different material like plastics, the color of the ball and the grass here is rather similar, so that a proper discrimination of the ball from the grass background is hard. A similar situation can occur to radar imaging when a target is located in a cluttered background. Figure 6 right shows average normalized RCS values of different terrain in dependence of incidence angle for two useful frequencies in landmine detection. Assuming now landmines or UXO as spherical and mostly dielectric objects, their RCS levels can be approximated by metallic or dielectric spheres of similar size. For those objects, the RCS can be computed and normalized values around $10^{-3}-10^{-4}$ or even lower can be obtained at those frequencies. Hence, the signature strength of such objects is in the same order as terrain RCS in the interesting incidence angle range of around $20-60^{\circ}$. This circumstance also makes clear that lower incidence angles are not useful due to strongly increasing background clutter, and higher incidence angles are less useful since the penetration capability into the ground decreases, and due to longer ranges, the signal-to-noise ratio becomes unacceptable. However, even in the useful range of incidence angles the discrimination of target signatures from clutter can be a serious problem.
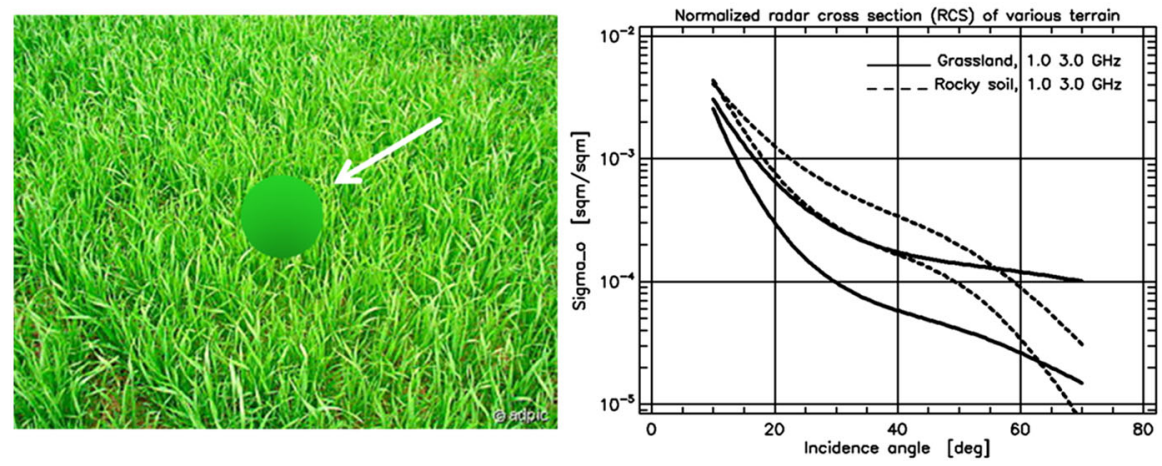

Fig. 6 Left photograph of a green ball located on green grass. Ball position is indicated by the white arrow. Right normalized RCS of various terrain types for two frequencies and vertical TX and RX polarization, based on a semi-empirical model from Ulaby (1989) (color figure online) 


\section{Philosophy and Design of the Radar System}

Following previous considerations, a SAR system using multiple channels, different polarization combinations, and operating in the UHF range was developed. Next the main features and their main drivers are briefly outlined:

\subsection{SAR Principle and Spatial Resolution}

SAR is an imaging principle based on a side-looking geometry, hence the radar can move on safe ground, while imaging the hazardous ground. Perpendicular to the linear radar motion, i.e., across track, narrow radar pulses are used to scan the area of interest. The delays of the pulses correspond to the range in across track direction. The width of the pulses, i.e., the bandwidth of the used signal, determines the resolution in time and consequently the range resolution via the knowledge of the speed of light. In the direction of radar motion, i.e., along track, the spatial resolution is achieved by coherently superimposing the radar echoes for a certain path length, called the synthetic aperture. By that way a large antenna is synthesized by the radar motion, similar to a real-aperture antenna of that size being not useful in practice due its size and mass. The size of the image is determined by the minimum and maximum across track distance to be scanned, and the length of the synthetic aperture.

\subsection{UHF Range}

Due to penetration depth constraints the frequency range should be lower than a few GHz. To use reasonable antenna sizes, the lowest frequency should be at least in the order of few $100 \mathrm{MHz}$.

\subsection{Multiple Channels}

The constraints of very limited target-to-clutter ratios to be expected for typical landmine/UXO scenarios require methods to improve that situation. From experience it was expected that a man-made target as a landmine typically shows in average similar signature strengths, even when incidence angle, bistatic angle, and aspect angle of observation are changed considerably. The required variability of angles can be achieved by high-resolution SAR, i.e., a large range of aspect angles for producing the synthetic aperture, and the use of multiple transmitters and receivers located on different positions in elevation. The latter produces on one hand different incidence angles for each TX/RX combination, and on the other hand, different bistatic angles when the average distance to the area of interest is sufficiently close to the radar. If furthermore the antenna arrangement for TX and $\mathrm{RX}$ is made such that the antenna array produces certain length in vertical direction, additionally vertical spatial resolution can be achieved, thus allowing a kind of three-dimensional imaging. In contrast to man-made targets, the natural background clutter usually is composed of multiple reflections within a resolution cell. Hence, their coherent superposition within the resolution cell changes quickly with different observation angles. In summary, by superposition of radar images from different 
observation angles the clutter is averaging out more than the man-made objects, producing a net gain in target-to-clutter ratio.

Following previous reasoning, the TIRAMI-SAR radar system was developed and constructed within the TIRAMISU Project, partly funded by Framework Programme 7 of the European Union (TIRAMISU Project 2012). A photograph of the final system and its main technical characteristics are given in Fig. 7. The radar was constructed using most modern radio frequency and digital electronics. Two independent TX channels and four independent RX channels deliver eight independent SAR images. Arbitrary signals can be generated fully digitally, e.g., a chirp or random waveforms, and on receive the full bandwidth is sampled for each RX channel directly at radio frequency in time domain. Hence, the maximum flexibility in signal processing is guaranteed and no information is lost due to analog pre-processing. The radar is completely mounted on a small truck. The antenna array is fixed to a rotatable boom to adjust the average incidence angle. The boom can be additionally elevated in vertical direction by a lifter. Thus, the array can be adjusted for optimum scene coverage and optimum RCS behavior. The single antennas have been mounted in the shown configuration where the top and bottom antennas are used for TX and the others for RX. This configuration has shown to be

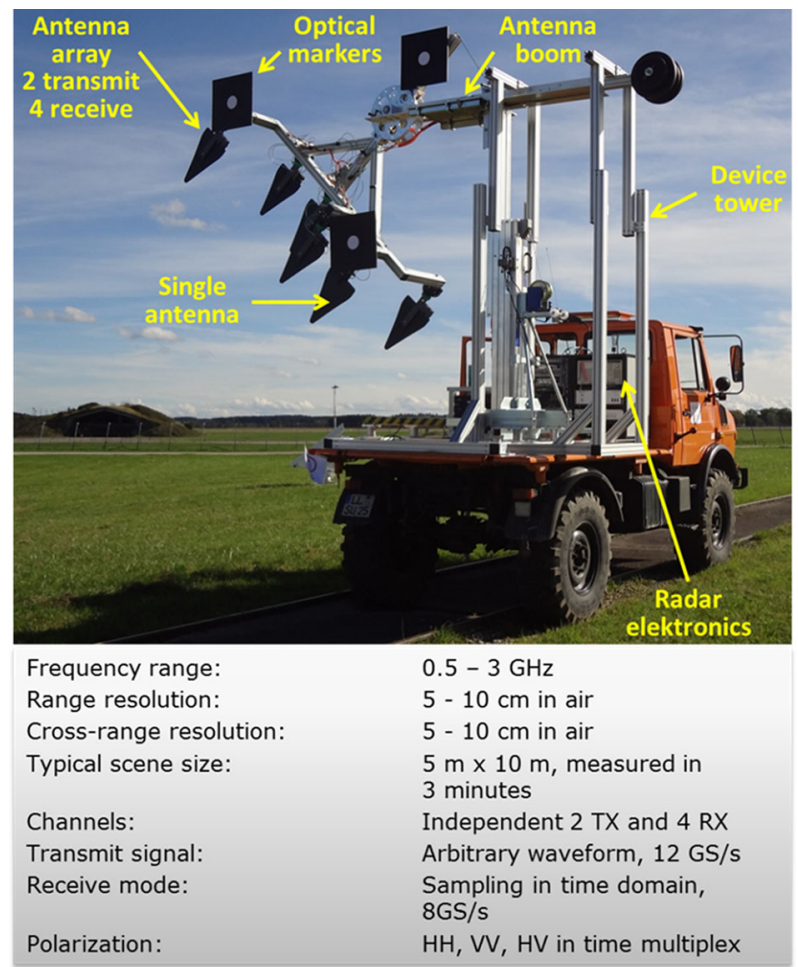

Fig. 7 Photograph of final TIRAMI-SAR radar system; below most important technical characteristics are given; $G S / s$ GigaSamples per second, $H$ horizontal $V$ vertical polarization, indication given for TX$\mathrm{RX}$. Optical markers are used for motion compensation of antenna motion with respect to the scene 
optimum with respect to observation angle variability and minimum cross talk between TX and RX antennas. Polarization configurations are adjusted by rotating single antennas manually to horizontal or vertical orientation. Hence, the different polarization combinations for TX-RX, i.e., $\mathrm{HH}, \mathrm{VV}$, and $\mathrm{HV}$, are measured one after the other for the identical scene. The SAR processing is currently done offline after the measurements. More information on TIRAMI-SAR and data processing is given in Schreiber et al. (2016) and Heinzel et al. (2016).

\section{Representative Measurement Results}

For a proof of concept and to verify proper SAR processing and error correction algorithms, various experiments on well-defined scenes have been carried out using a sandbox at DLR facilities in Oberpfaffenhofen. For instance, an array of trihedral radar reflectors was located on the surface of the sand to verify proper focusing of the SAR processor all over the image area. A photograph of that scene and the corresponding SAR image for superposition of all TX-RX combinations are shown in Fig. 8. In the

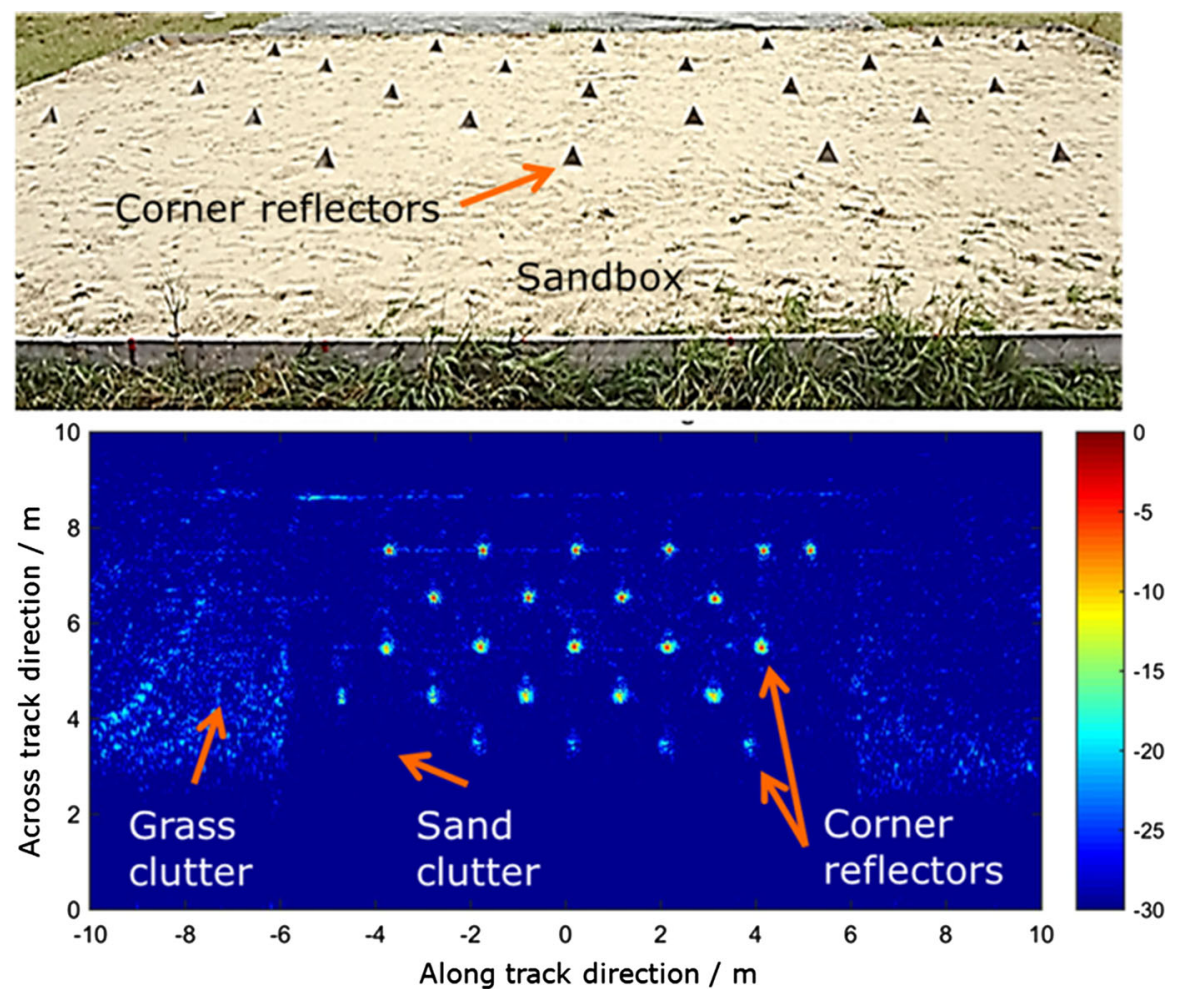

Fig. 8 Top photograph of an array of trihedral radar reflectors located on the surface of a sandbox. The sandbox has a size of $12 \mathrm{~m}$ length and $8 \mathrm{~m}$ width, and it is surrounded by grassland; bottom corresponding SAR image of that scene for superposition of all TX-RX combinations. The color bar is indicated in $\mathrm{dB}$, and the image is normalized to its maximum value (color figure online) 

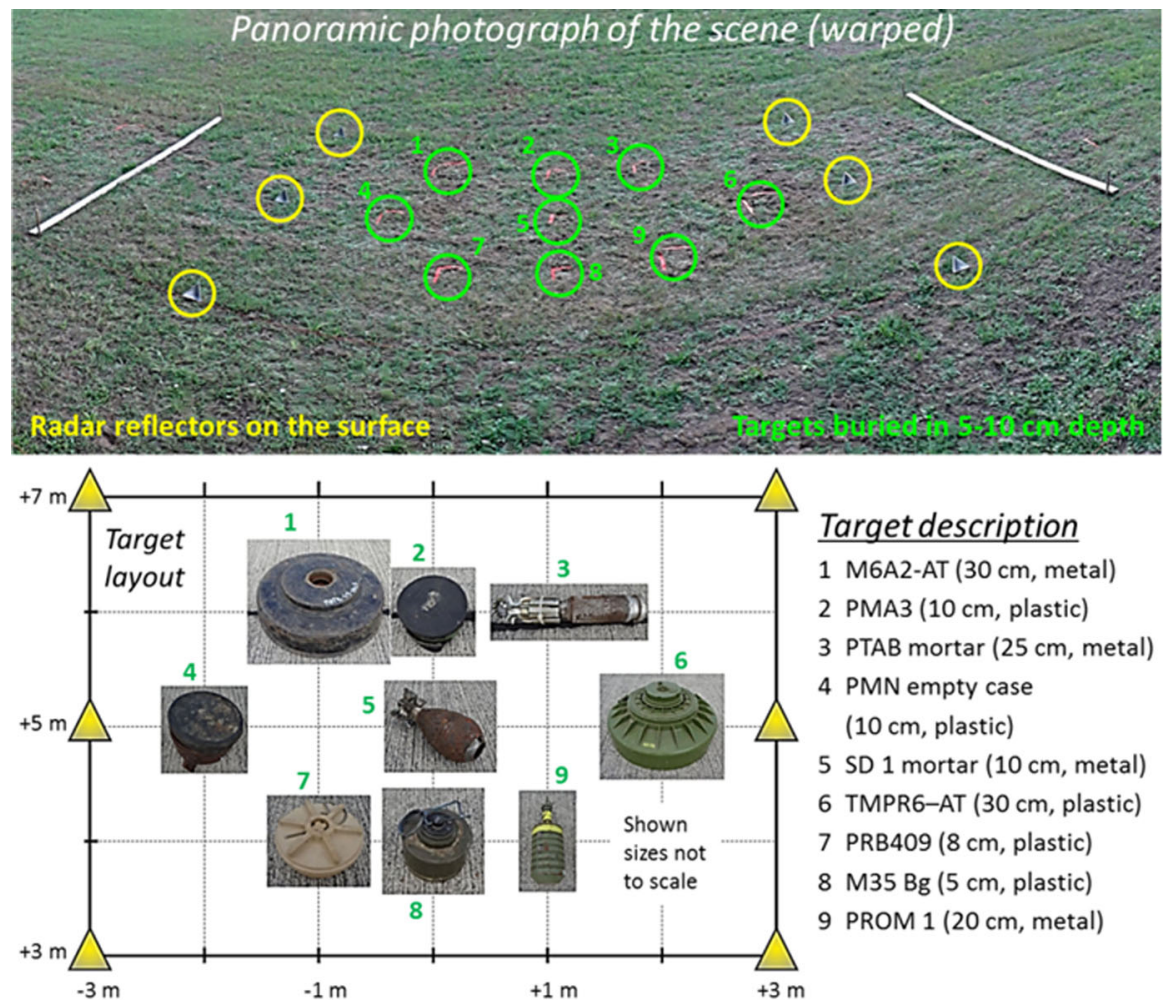

\section{Target description}

$1 \mathrm{M} 6 \mathrm{A2}-\mathrm{AT}$ ( $30 \mathrm{~cm}$, metal)

2 РMA3 (10 cm, plastic)

3 PTAB mortar $(25 \mathrm{~cm}$, metal)

4 PMN empty case

(10 cm, plastic)

5 SD 1 mortar $(10 \mathrm{~cm}$, metal)

6 TMPR6-AT ( $30 \mathrm{~cm}$, plastic)

7 PRB409 (8 cm, plastic)

$8 \mathrm{M} 35 \mathrm{Bg}$ (5 cm, plastic)

9 PROM 1 (20 cm, metal)

Fig. 9 Top photograph of a buried object scene. The surface is grassland on conventional soil. Indicated are the locations of the buried targets and some trihedral radar reflectors located on the surface as reference targets; bottom photographs and positions of all targets. Additionally, names, diameter and core material of the buried targets are given

SAR image, the radar reflectors are clearly visible as point sources showing correct focusing all over the image. They have a relatively high normalized RCS of about 0.05 compared to the background. It should be considered that flat sand has lower clutter level than the terrain materials shown in Fig. 6, e.g., $\sigma^{0} \leq 0.0001$. Hence, the observed reflector-to-clutter ratio can be up to $30 \mathrm{~dB}$ as being observed in the radar image. However, it can be as well recognized that the adjacent grass land shows considerably higher clutter level of up to $10 \mathrm{~dB}$ more than the sand area.

Next a landmine/UXO scene is discussed. Here, several typical objects have been buried in normal soil covered by grass. That setup was provided during a TIRAMISU measurement campaign at DOVO/SEDEE in Leuven, Belgium. A photograph of the grass surface together with photographs of the targets and the indications of their location is shown in Fig. 9. Figure 10 shows the single SAR image for one TX-RX and one polarization combination. It is evident that the radar reflectors again can be clearly identified as a focused point source, but in the buried target area no clear discrimination of target and clutter responses can be performed. That observation fully corresponds to the theoretical considerations outlined in 


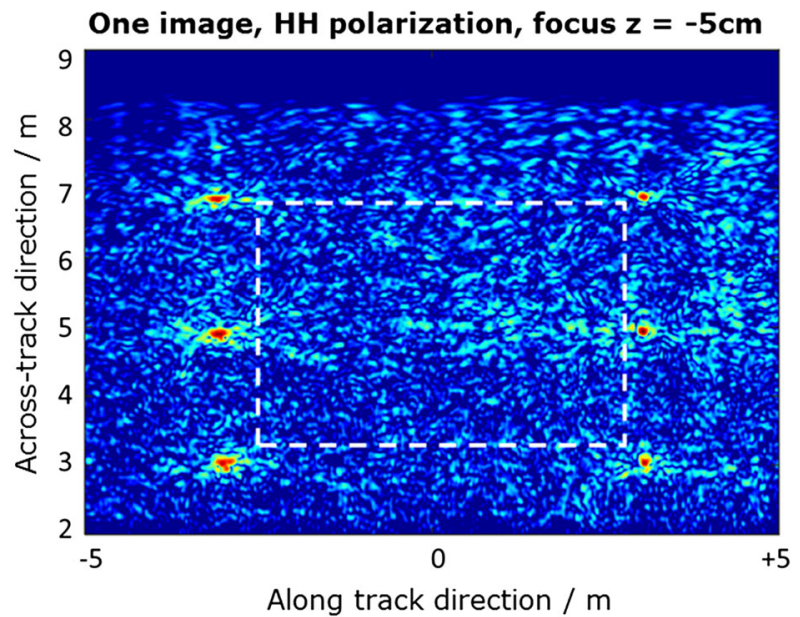

Fig. 10 SAR image of the scene described in Fig. 9 for only one TX-RX combination in HH polarization. The focusing plane was set to $-5 \mathrm{~cm}$ below the reference level of the surface. The dashed rectangle indicates the area where the targets have been buried

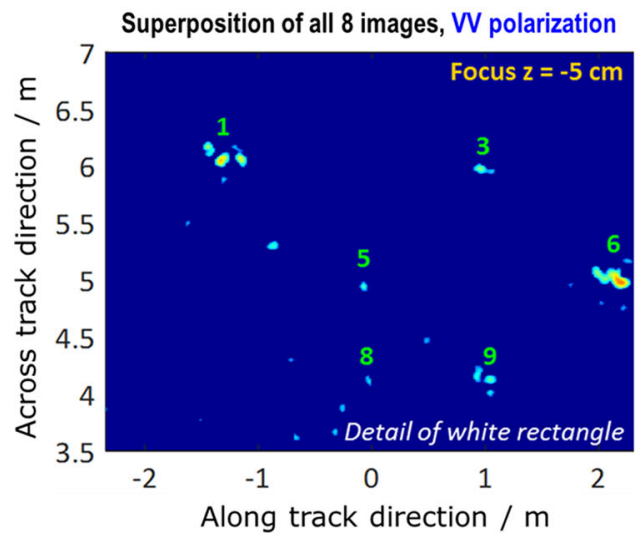

Superposition of all 8 images, $\mathrm{HH}$ polarization

Fig. 11 Superimposed SAR images for all eight antenna combinations; left VV polarization; right $\mathrm{HH}$ polarization. The image size corresponds to the rectangular area of Fig. 10 enclosed by the white dashed line

Sect. 2.4, and thus proper object detection can fail in case of methods based on analyzing only single SAR images. Here the TIRAMI-SAR approach using superposition of multi-static and multi-polarization information demonstrates its power. Figure 11 now illustrates the superposition of all eight SAR images acquired by $2 \times 4$ antenna combinations for $\mathrm{HH}$ and VV polarization combination.

Now the buried targets can be clearly discriminated from the clutter. While targets 2 and 7 are detected for $\mathrm{HH}$ polarization they are not for VV polarization. Only target 4 is missing in both images, probably due to the fact that the mine was only simulated by an empty case for which the wall thickness is quite small 
compared to the wavelengths. However, this result shows the importance and necessity of precisely superimposing several SAR images of different observation angles and using polarimetric information. This statement could be verified by various other experiments showing similar results.

\section{Conclusions}

A novel radar approach has been developed and applied to the problem of reliable landmine and UXO detection at high throughput of scanned area per time. This method allows detection and localization of buried and surface objects in the presence of background clutter, while up to now no discrimination of objects concerning a threat or a false alarm is performed. Moreover, the philosophy of the concept is to perform first reliable and effective detection of suspicious objects, and then to use other sensors based on different physical principles to investigate further those locations where detections occurred. By that way the overall system acts as a multi-sensor system, being the only successful approach to face and solve the problem for our opinion.

The use of radar for buried object detection is based on the measurement of bulk material anomalies, producing mismatch of wave impedance, and thus giving rise for wave scattering and reflections. Microwave radar thus is not sensitive to spectroscopic properties, and thus cannot discriminate explosives from other matter just by signature. Consequently, the multi-sensor approach as mentioned above will do the discrimination.

The use of SAR technology provides the unique benefits of combining sidelooking geometry for operation from safe area into hazardous area, high area throughput due to large image size, and highest spatial resolution for detection of even small and weak objects in RCS. The problem of background clutter is solved by applying a multi-channel system in TX and RX, providing different observation angles for each TX-RX combination. In addition, the use of wave polarization improves further considerably detection performance.

Although TIRAMI-SAR already performs satisfying at this stage, it offers plenty of room for further optimization and improvements. Especially, the use of other antenna array geometries, other waveforms, other polarization combinations, etc., is of major interest. Furthermore, many other experiments and field trials have to be executed to make performance assessment more reliable and informative.

Acknowledgements Funding was provided by EU Framework Programme 7 (FP7-SEC-2011-1 No. 284747).

\section{References}

Feng X et al (2005) Estimation of ground surface topography and velocity model by SARGPR and its application to landmine detection. In: SPIE conference detection and remediation for mines and minelike targets X, Vol. 5794

Globalgiving (2016). https://www.globalgiving.org/projects/mli-mine-detection-dogs/. Accessed 27 July 2016 
Heinzel A et al (2016) Focusing methods for ground penetrating MIMO SAR Imaging within half-spaces of different permittivity. In: Proceedings of 11th European conference on synthetic aperture radar EUSAR'16, pp 842-846

Kabourek V et al (2012) Clutter reduction based on principal component analysis technique for hidden objects detection. Radioengineering 21(1):464-470

Landmine Monitor (2015). http://www.the-monitor.org/en-gb/reports/2015/landmine-monitor-2015/ preface.aspx. Accessed 27 July 2016

Lawrence C et al (1999) Ultra-wide-band synthetic aperture radar for mine-field detection. IEEE Antennas Propag Mag 41(1):18-33

Liu $G$ et al (2003) SAR imaging for a forward-looking GPR system, In: SPIE conference detection and remediation for mines and minelike targets VIII, Vol. 5089

Lloyd D, Longstaff I D (2003) Ultra-wideband multistatic SAR for the detection and location of landmines. IEE proceedings. Online No. 20030444

Pica.Army (2016). http://www.pica.army.mil/Picatinny/products_services/products15.aspx. Accessed 27 July 2016

Schreiber E et al (2016) Challenges for operational use of ground-based high-resolution SAR for landmines and UXO detection. In: Proceedings of 11th European conference on synthetic aperture radar EUSAR'16, pp 834-837

Telovation (2016). http://www.telovation.com/articles/land-mine-detection-robot.html. Accessed 27 July 2016

Theleadsouthaustralia (2016). http://www.theleadsouthaustralia.com.au/industries/defence/minelab-tosupply-us-military-with-mounted-mine-detection-system/. Accessed 27 July 2016

TIRAMISU Project (2012) Project website. http://fp7-tiramisu.eu. Accessed 27 July 2016

Ulaby FT (1981) Microwave remote sensing-active and passive. Microwave remote sensing fundamentals and radiometry, vol I. Addison-Wesley, Reading

Ulaby FT (1982) Microwave remote sensing-active and passive. Radar remote sensing and surface and emission theory, vol II. Addison-Wesley, Reading

Ulaby FT (1989) Handbook of radar scattering statistics for terrain. Artech House, Norwood

Ulaby FT (1992) Modelling radar backscatter from vegetation. Short course notes. DLR, Oberpfaffenhofen

Wu H et al (2010) Motion compensation for landmine detecting vehicle-borne SAR. In: Proceedings of IEEE 11th international radar symposium IRS'10 